\title{
ABSENTEÍSMO NA EQUIPE DE ENFERMAGEM EM UNIDADES DE CUIDADOS CRÍTICOS: UMA REVISÃO INTEGRATIVA
}

\author{
Bruno Del Sarto AZEVEDO ${ }^{1}$ \\ ${ }^{1}$ Enfermeiro. Mestrando do Programa de Pós-graduação em Enfermagem e Saúde da Universidade Estadual do \\ Sudoeste da Bahia. E-mail: brunodelsarto@outlook.com
}

Recebido em: 30/05/2014 - Aprovado em: 25/09/2014 - Disponibilizado em: 15/12/2014

\begin{abstract}
RESUMO: Este estudo objetivou identificar o que a literatura científica tem abordado em relação ao absenteísmo na equipe de enfermagem no contexto das unidades hospitalares de terapia intensiva e de atendimento de urgência e emergência. Realizou-se a busca nas bases de dados contidas na Biblioteca Virtual em Saúde, Pubmed, Scopus e Web of Science. Os termos utilizados foram "absenteeism" e "nursing", resultando na seleção de 6 artigos. Os achados desta revisão possibilitam um vislumbre sobre a problemática do absenteísmo entre a equipe de enfermagem lotadas nas unidades em questão, destacando-se a maior associação de ausências não previstas entre o pessoal de nível médio e entre aqueles que trabalham à noite. A principal causa das ausências foi o adoecimento, sobretudo por distúrbios osteomusculares e transtornos mentais e comportamentais. Ressaltam-se ainda os efeitos negativos do absenteísmo sobre a organização e a qualidade da assistência prestada ao paciente. Contudo, devido ao delineamento metodológico dos artigos desta revisão, tais achados não fornecem evidência conclusiva sobre esse fenômeno nos setores em questão. Sendo assim, pesquisas futuras devem ser conduzidas, de modo a orientar o desenvolvimento de intervenções que visem resolver este problema, a fim de promover impacto positivo sobre a qualidade de vida no trabalho desses trabalhadores e de melhorar a qualidade da assistência de enfermagem.
\end{abstract}

Palavras-chave: Absenteísmo. Equipe de enfermagem. Unidades de terapia intensiva. Serviços médicos de emergência. Saúde do trabalhador.

\section{ABSENTEEISM AMONG NURSING TEAM IN CRITICAL CARE UNITS: AN INTEGRATIVE REVIEW}

\begin{abstract}
This study aimed to identify what the scientific literature has addressed in regard to absenteeism in the nursing team in the context of hospital intensive care units and urgency/emergency services. A search was conducted in the databases of the Virtual Health Library, PubMed, Scopus and Web of Science. The used terms were "absenteeism" and "nursing", resulting in the selection of six scientific papers. The findings allowed a glimpse on the absenteeism problem among the nursing team working in these units. We highlight the association of non-predicted absences among the nursing assistants and workers who labors at night. The major causes of these absences were sickness, especially musculoskeletal and mental/behavioral disorders. We also emphasize the negative effects of absenteeism on the organization and quality of patient care. However, due to the methodological design of the analyzed papers, these findings do not provide conclusive evidence on this phenomenon in the considered sectors. Thus, future research should be conducted to guide the development of interventions with the purpose to solve this problem and promote positive impact on the quality of working life of these workers and on the quality of nursing care.
\end{abstract}

Keywords: Absenteeism. Nursing, Team. Intensive Care Units. Emergency Medical Services. Occupational Health. 


\section{INTRODUÇÃO}

O absenteísmo, absentismo ou ausentismo, entendido como as ausências no trabalho do pessoal, vem se apresentando como um sério problema para as organizações e seus gerentes, dada sua origem multifatorial (LIMA; KURGANCT, 2009; GAUDINE et al., 2013).

Pesquisa canadense revelou que, em 2006, o trabalhador de saúde com idade entre 25 e 54 anos de idade, perdeu quase 12 dias de trabalho em decorrência de doença ou incapacidade, comparados a 7 dias de trabalho perdidos pelas pessoas ocupadas em outros setores. O problema é ainda mais evidente para a categoria de enfermagem, que, entre as profissões da área da saúde, teve a maior média de dias perdidos decorrentes de doença ou incapacidade, com 14 dias perdidos, quase o dobro da média para todas as ocupações (STATISTICS CANADA, 2007).

Nos últimos anos, diversas mudanças nos cuidados de saúde a nível internacional têm aumentado a carga de trabalho da equipe de enfermagem. Incluem-se, por exemplo, o aumento no número de pacientes e de suas necessidades, a diminuição no tempo de internação, a relativa falta de recursos econômicos, a busca constante por melhor produtividade e o aumento da competição na área da saúde (RAUHALA et al., 2007; MAGALHÃES; DALL'AGNOL; MARCK, 2013).
Além da elevada carga de trabalho, outros fatores de risco ocupacional podem se caracterizar como geradores de absenteísmo para a equipe de enfermagem, tais como o ambiente e materiais de trabalho inadequados, equipamentos de proteção individual e coletiva insuficientes e/ou inadequadamente utilizados, postura corporal inadequada na realização das tarefas e ritmo de trabalho acelerado pela falta de recursos material e humano (DUARTE; MAURO, 2010).

Há evidências, ainda, de que o absenteísmo está associado também a problemas de segurança do paciente (como o aumento da incidência de quedas do leito e de infecções associadas ao cateter venoso central), através do aumento da carga de trabalho e estresse dos enfermeiros presentes no trabalho (RAUHALA et al., 2007).

Tal problemática mostra-se ainda mais visível e complexa em unidades hospitalares destinadas à assistência de pacientes críticos, como as Unidades de Terapia Intensiva (UTI) e os Prontos-Socorros (PS), uma vez que os cuidados e recursos tecnológicos requerem equipe qualificada, o que torna difícil a substituição em casos de ausência não prevista.

Diversos estudos têm evidenciado que o absenteísmo entre as equipes de enfermagem lotadas em UTI e PS, sejam estes destinados à assistência a adultos, crianças ou neonatos, está entre os mais altos dentro do contexto hospitalar (COSTA; VIEIRA; 
SENA, 2009; SANCINETTI et al., 2009; ABREU; SIMÕES, 2009).

Por essa razão, reconhecendo a importância de se ter um melhor entendimento sobre essa problemática dentro das referidas unidades, este estudo objetivou identificar o que a literatura científica tem abordado em relação ao absenteísmo na equipe de enfermagem no contexto das unidades de terapia intensiva e de atendimento de urgência e emergência.

\section{METODOLOGIA}

Trata-se de uma revisão integrativa, realizada em junho de 2013, por meio de consulta às bases de dados contidas na Biblioteca Virtual em Saúde (BVS), Pubmed, Scopus e Web of Science. Os termos utilizados para a busca foram "absenteeism" e "nursing", selecionados a partir dos Descritores em Ciências da Saúde (DeCS) e do Medical Subject Headings (MeSH). Operou-se o rastreamento através da utilização do operador booleano "AND" entre os descritores supracitados.

Foram estabelecidos como critérios de inclusão: ser documento do tipo artigo científico, com disponibilidade de texto completo; ter sido publicado entre os anos de 2008 e 2012; estar nos idiomas português, inglês ou espanhol; abordar, de forma central, a temática do absenteísmo na equipe de enfermagem; e ter como local de estudo UTI ou unidade de atendimento de urgência e emergência (PS).

Tal busca identificou um total de 560 achados, nos quais foram excluídos aqueles que se repetiam em mais de uma base de dados. Foi realizada, então, leitura criteriosa dos títulos, selecionando, assim, 50 artigos científicos. Estes, por sua vez, tiveram seus resumos lidos na íntegra e passaram por uma leitura flutuante de todo o corpo do estudo, a fim de identificar quais atendiam aos critérios de inclusão do presente estudo. Ao final desta etapa, foram selecionados 6 artigos, julgados coerentes com a proposta desta revisão.

Tais estudos foram lidos, caracterizados e fichados, de modo a compilar, dentre outras variáveis: título do artigo, identificação dos autores e do periódico, ano de publicação, objetivo, população do estudo, bem como os principais resultados e conclusões. A partir daí, procedeu-se a avaliação e interpretação dos resultados, os quais foram sintetizados e organizados, de modo a orientar a discussão.

\section{RESULTADOS E DISCUSSÃO}

Dos seis estudos selecionados, quatro tiveram como local de estudo UTI e dois PS. Todos eles são pesquisas de âmbito nacional, sendo três deles investigações no Paraná, dois em São Paulo e um na Bahia.

Não foram encontrados estudos internacionais que tiveram como local de 
pesquisa estas unidades especificamente, apresentando a maioria deles investigações sobre todo o contex to hospitalar.
Segue abaixo o Quadro 1, apresentando uma síntese das principais características, resultados e conclusões dos estudos selecionados nesta revisão.

Quadro1 - Principais características e achados dos artigos selecionados do estudo. Jequié-BA, Brasil, 2013.

\begin{tabular}{|c|c|c|c|}
\hline $\mathbf{N}^{\mathbf{o}}$ & $\begin{array}{l}\text { Autores, ano de } \\
\text { publicação e } \\
\text { periódico }\end{array}$ & Objetivo(s) & Principais resultados e conclusões \\
\hline 1. & $\begin{array}{l}\text { FAKIH; } \\
\text { TANAKA; } \\
\text { CARMAGNANI, } \\
2012 . \\
\text { Acta Paulista de } \\
\text { Enfermagem }\end{array}$ & $\begin{array}{l}\text { Verificar e analisar as } \\
\text { ausências previstas e não } \\
\text { previstas dos } \\
\text { colaboradores r de } \\
\text { enfermagem da unidade } \\
\text { de pronto-socorro. }\end{array}$ & $\begin{array}{l}\text { O PS de adultos teve, em média, } 96,8 \text { colaboradores por mês. } \\
\text { A taxa de absenteísmo foi de } 11,7 \% \text {. Houve maior incidência } \\
\text { de ausências não previstas no período de maio }(15,3 \%) \text { e } \\
\text { agosto (13,3\%). Houve correlação entre as variáveis: } \\
\text { categoria profissional, vínculo empregatício e turno de } \\
\text { trabalho e a distribuição das ausências previstas e não } \\
\text { previstas. O absenteísmo foi considerado elevado e motivado, } \\
\text { sobretudo, pelas licenças para tratamento de saúde superiores } \\
\text { a } 15 \text { dias. O déficit mensal de pessoal contribuiu também } \\
\text { para a sobrecarga do trabalho da equipe. }\end{array}$ \\
\hline 2. & $\begin{array}{l}\text { CARNEIRO; } \\
\text { FAGUNDES, } \\
2012 . \\
\text { Revista } \\
\text { Enfermagem UERJ }\end{array}$ & $\begin{array}{lr}\text { Levantar as } & \text { taxas de } \\
\text { absenteísmo } & \text { entre } \\
\text { trabalhadoras } & \text { em } \\
\text { enfermagem e definir o } \\
\text { índice de } & \text { segurança } \\
\text { técnica (IST) que melhor } \\
\text { se ajusta à realidade } \\
\text { pesquisada. }\end{array}$ & $\begin{array}{l}\text { Durante o ano de } 2009 \text {, foram registrados } 336 \text { dias perdidos } \\
\text { de trabalho no grupo de } 23 \text { enfermeiras e } 548 \text { dias no grupo } \\
\text { das } 41 \text { auxiliares e técnicas de enfermagem, perfazendo } 884 \\
\text { dias no total. O afastamento por doenças foi elevado tanto } \\
\text { para enfermeiras quanto para técnicas e auxiliares. As taxas } \\
\text { de ausências entre as enfermeiras variaram entre } 8 \% \text { e } 30 \% \text { e } \\
\text { das auxiliares e técnicas de enfermagem, entre } 12 \% \text { e } 46 \% \text {. } \\
\text { Concluiu-se que, entre as auxiliares e técnicas, essa taxa foi } \\
\text { elevada, sobrepondo-se ao IST de } 30 \% \text { utilizado na } \\
\text { organização. }\end{array}$ \\
\hline 3. & $\begin{array}{l}\text { CAMPOS; } \\
\text { JULIANI; } \\
\text { PALHARES, } \\
2009 . \\
\begin{array}{l}\text { Revista Eletrônica } \\
\text { de Enfermagem }\end{array}\end{array}$ & $\begin{array}{l}\text { Investigar o real número } \\
\text { de ausências dos técnicos } \\
\text { e auxiliares de } \\
\text { enfermagem de um PS de } \\
\text { um Hospital Universitário } \\
\text { do interior do estado de } \\
\text { São Paulo para definir o } \\
\text { IST. }\end{array}$ & $\begin{array}{l}\text { No período investigado, cerca de } 70 \% \text { das ausências se } \\
\text { trataram de ausências previstas. Entre as não previstas } \\
\text { destacaram-se as licenças médicas, que representou algo em } \\
\text { torno de } 25 \% \text { do total de ausências e que podem estar } \\
\text { relacionadas à sobrecarga da equipe. Encontrou-se IST cuja } \\
\text { média gira em torno de } 51 \% \text {. }\end{array}$ \\
\hline 4. & $\begin{array}{l}\text { INOUE; } \\
\text { MATSUDA; } \\
\text { SILVA, } 2008 . \\
\text { Ciência, Cuidado e } \\
\text { Saúde. }\end{array}$ & $\begin{array}{lr}\text { Verificar o índice } & \text { de } \\
\text { absenteísmo } & \text { dos } \\
\text { trabalhadores } & \text { da } \\
\text { enfermagem da UTI. } & \end{array}$ & $\begin{array}{l}\text { Constatou-se que o índice de absenteísmo (IA) da equipe de } \\
\text { enfermagem da UTI para adultos }(2,55 \%) \text { foi baixo, e a } \\
\text { principal causa de ausência não programada do funcionário } \\
\text { ao trabalho foi o absenteísmo-doença }(66,95 \%) \text {. Houve maior } \\
\text { IA entre o pessoal de enfermagem de nível médio }(2,91 \%) \text { do } \\
\text { que entre os enfermeiros }(1,82 \%) \text {. Concluiu-se que, apesar de } \\
\text { o IA ter sido baixo, os trabalhadores de enfermagem estão } \\
\text { sobrecarregados pelo número reduzido de pessoal. }\end{array}$ \\
\hline 5. & $\begin{array}{l}\text { INOUE et al., } \\
2008 . \\
\begin{array}{l}\text { Revista Brasileira } \\
\text { de Enfermagem }\end{array}\end{array}$ & $\begin{array}{l}\text { Investigar o absenteísmo- } \\
\text { doença na UTI para } \\
\text { adultos de um hospital- } \\
\text { escola do Paraná. }\end{array}$ & $\begin{array}{l}\text { A maioria dos trabalhadores é do sexo feminino }(76,8 \%) \text {, } \\
\text { com } 30 \text { a } 39 \text { anos }(42,9 \%) \text {, com vínculo estatutário }(66,1 \%) \text {. } \\
\text { A média de dias de trabalho perdida foi maior para os } \\
\text { trabalhadores de nível médio }(2,9) \text {, do sexo feminino }(3,2) \text {, } \\
\text { com vínculo temporário }(4,4) \text { e do período noturno }(6,2) \text {. O } \\
\text { absenteísmo-doença foi considerado elevado. }\end{array}$ \\
\hline 6. & CASTRO; & Descrever o perfil dos & Os auxiliares de enfermagem foram responsáveis pelo maior \\
\hline
\end{tabular}




\begin{tabular}{|c|c|c|}
\hline $\begin{array}{l}\text { BERNARDINO; } \\
\text { RIBEIRO, } 2008 . \\
\text { Cogitare } \\
\text { Enfermagem }\end{array}$ & $\begin{array}{l}\text { trabalhadores implicados } \\
\text { no absenteísmo da UTI } \\
\text { neonatal e identificar os } \\
\text { motivos das ausências não } \\
\text { previstas desses } \\
\text { trabalhadores }\end{array}$ & $\begin{array}{l}\text { número de afastamentos ( } 73 \%) \text {, com idade entre } 31 \text { a } 40 \text { anos } \\
(47 \%) \text {, casada }(68 \%) \text {, com filhos }(68 \%) \text { e com um emprego } \\
(76 \%) \text {. Os motivos mais observados foram licença para } \\
\text { cuidar da própria saúde }(44 \%) \text { e para cuidar de familiar } \\
(32 \%) \text {. }\end{array}$ \\
\hline
\end{tabular}

\section{Determinantes do absenteísmo entre a equipe de enfermagem}

Somente um estudo apresentou associação entre o sexo e o absenteísmo, verificando relação diretamente proporcional entre ser mulher e se ausentar mais do trabalho (INOUE et al., 2008). A maior ausência no trabalho entre as mulheres pode ser explicada pela frequente conciliação do exercício profissional, por vezes em mais de um local, com atividades domésticas, o que representa desgaste físico e mental que, cedo ou tarde, pode repercutir em adoecimento (BARBOZA; SOLER, 2003).

Quanto à faixa etária que apresenta maiores taxas de absenteísmo, os estudos mostraram grande variação (21 a 49 anos), não havendo, portanto, consenso quanto a esta característica (CASTRO; BERNARDINO; RIBEIRO, 2008; INOUE et al., 2008). Apenas um estudo (CASTRO; BERNARDINO; RIBEIRO, 2008) relacionou as variáveis situação conjugal e número de filhos à taxa de absenteísmo, apontando uma maioria de casados e de trabalhadores com filhos entre aqueles que mais se ausentam do trabalho, o que está também associado à execução das atividades domésticas no âmbito familiar, ficando responsável pelo cuidado com a casa e pela educação dos filhos, o que pode gerar desgaste e, consequentemente, absenteísmo. O dado sobre o estado marital corrobora com outro estudo realizado entre profissionais de enfermagem de três hospitais públicos (FERREIRA et al., 2012).

Com relação à variável número de empregos, esta não mostrou associação com maior impacto nas ausências (CASTRO; BERNARDINO; RIBEIRO, 2008). O tipo de vínculo empregatício também mostrou achados divergentes, mostrando maioria de ausências tanto entre os trabalhadores estatutários $\quad$ FAKIH; $\quad$ TANAKA; CARMAGNANI, 2012), quanto entre os celetistas (INOUE et al., 2008).

Os estudos mostraram convergência quanto à associação positiva entre trabalho noturno e absenteísmo (INOUE et al., 2008; FAKIH; TANAKA; CARMAGNANI, 2012). A desregulação do ritmo circadiano pode acarretar alterações nos mais diversas funções corpóreas, podendo ocasionar prejuízos à saúde do trabalhador e com isso levar a um maior número de ausências não previstas do trabalho (IZU et al., 2011). 
Cinco dos seis estudos tiveram como população/amostra tanto enfermeiros como técnicos e auxiliares de enfermagem. Em todos (INOUE et al., 2008; INOUE; MATSUDA; SILVA， 2008; CASTRO; BERNARDINO; RIBEIRO, 2008; CARNEIRO; FAGUNDES，2012; FAKIH; TANAKA; CARMAGNANI, 2012) verificou-se um maior índice de absenteísmo entre o pessoal de nível médio. Pode-se explicar tal resultado pela própria competência do cargo de enfermeiro, que possui maiores responsabilidades, condicionando sua presença mais constante ao trabalho, mesmo quando debilitado física e mentalmente (INOUE et al., 2008; INOUE; MATSUDA; SILVA, 2008). Ou ainda pelo fato de que os grupos mais qualificados, com formação superior, estão menos expostos às más condições de trabalho, resultando em menor adoecimento e absenteísmo (FAKIH; TANAKA; CARMAGNANI, 2012).

Todos os estudos revelam o afastamento por doença como o principal motivo das ausências não previstas. Porém, apenas um estudo (FAKIH; TANAKA; CARMAGNANI, 2012) investiga as causas fisiológicas e psicológicas constantes nas licenças para tratamento de saúde. As principais são as doenças do sistema osteomuscular e do tecido conjuntivo e os transtornos mentais e comportamentais.

Estudo de Ferreira et al. (2012) revelou que entre aqueles profissionais de enfermagem que referiram mais de duas doenças osteomusculares, a chance de referir de 1 a 9 dias de ausência foi cerca de duas vezes mais elevada, além de três vezes mais elevada de referir 10 dias ou mais de ausência.

No que diz respeito aos transtornos mentais e comportamentais, na ausência de reconhecimento, autonomia e poder, os enfermeiros veem suas responsabilidades não como fonte de orgulho profissional, mas como fonte de ansiedade (ALDERSON, 2008). Esta realidade, aliada à sobrecarga de trabalho e aos conflitos dentro da própria equipe (de enfermagem e multiprofissional), possibilita o surgimento ou agravamento dessa condição.

Inclui-se nesse grupo de transtornos a Síndrome de Burnout. O desgaste proporcionado por esta síndrome se reflete em diminuição importante do rendimento no trabalho e consequentemente aumento de absenteísmo entre a equipe de enfermagem (DAVEY et al., 2009; CARVALHO; MAGALHÃES, 2011).

Outros estudos confirmam maior frequência de doenças osteomusculares e de transtornos mentais e comportamentais nos trabalhadores de enfermagem (SANCINETTI et al., 2009; ABREU; SIMÕES, 2009; MAGALHÃES et al., 2011).

Nesse sentido, as características do processo de trabalho desenvolvido por estes trabalhadores, que determinam sua exposição 
a diferentes cargas, são causadoras de desgaste físico e mental, ocasionando, muitas vezes, afastamento do trabalho por doença (SANCINETTI et al., 2011).

\section{Consequências e desafios impostos pelo absenteísmo}

Os estudos revisados consideram o absenteísmo como um problema para a qualidade da assistência prestada ao paciente e às condições laborais dos trabalhadores. Além disso, os dias de ausência constituem-se em situação que podem gerar necessidade de horas extra para cobertura de plantões e, com isto, mais custos para a organização.

Castro, Bernardino e Ribeiro (2008) chamam a atenção, ainda, para o fato de que quanto maior o período de afastamento, maior será o número de profissionais necessários para substituição, podendo acarretar prejuízos na assistência, além de sobrecarga de trabalho e desgaste dos demais integrantes da equipe.

Nessa perspectiva, em situação de crise, na qual se faz necessária contenção de recursos financeiros, ocorre diminuição do número de contratos. $\mathrm{O}$ efeito cascata que se produz é o de sobrecarga de trabalho, que leva ao aumento de faltas e afastamentos por doenças, sendo, portanto, questionável se esse tipo de medida administrativa reduz de fato os custos ou gera aumento dos mesmos em decorrência dos desdobramentos suscitados (CAMPOS; JULIANI; PALHARES, 2009).
Sendo assim, com ou sem crise, dificilmente há redução do número de leitos ou da carga de trabalho e o cliente precisa continuar sendo atendido diante de suas necessidades de saúde. Deste modo, a prestação de assistência humanizada e de qualidade depende, não somente, mas também das condições de trabalho a que está submetida a equipe de enfermagem (CAMPOS; JULIANI; PALHARES, 2009).

Gaudine et al. (2013) assinalam ainda outros obstáculos à redução do absenteísmo, dentre eles: a programação inflexível; o estresse no trabalho e em casa; e as condições de trabalho desmoralizantes, o que inclui baixos salários, baixa motivação no trabalho e dificuldades de comunicação com os colegas e com a gerência.

\section{Intervenções sugeridas para minimização do absenteísmo}

Primeiramente, tem-se a necessidade de a coordenação de enfermagem das unidades em questão manterem um olhar atento e contínuo sobre os índices de absenteísmo, como forma de se realizar um novo dimensionamento de pessoal, se necessário. Além disso, deve-se buscar o aprofundamento sobre a origem das doenças que estão levando ao afastamento dos trabalhadores, a fim de aplicar medidas que possam mitigá-las ou minimizá-las 
(CASTRO; BERNARDINO; RIBEIRO, 2008; CARNEIRO; FAGUNDES, 2012).

Outras medidas a serem tomadas a fim de minimizar a problemática do absenteísmo são: a solicitação da intervenção do serviço de saúde ocupacional para acompanhamento dos trabalhadores com problemas de saúde e prevenção de novos adoecimentos, além da promoção à saúde e segurança no trabalho; a proposição à direção da organização de mudanças no índice de segurança técnica, visando à uniformidade e avaliação posterior da qualidade da assistência, bem como de mudanças efetivas na política de gestão de pessoal e nos processos de trabalho; a realização, durante as reuniões das unidades, de encontros entre a equipe de enfermagem e a psicóloga do serviço de desenvolvimento de pessoas, cuja temática seja o relacionamento interpessoal e outros temas afins (CARNEIRO; FAGUNDES, 2012; (FAKIH; TANAKA; CARMAGNANI, 2012).

No que concerne às mudanças nas condições de trabalho na UTI ou PS, sugerese a utilização de medidas como: promoção de pausa para descanso durante cada plantão; criação de canais de escuta da opinião e sugestões dos trabalhadores sobre as condições para a execução da assistência na unidade, bem como de um programa de ginástica laboral durante o horário de trabalho (CARNEIRO; FAGUNDES, 2012).

$$
\text { Carneiro e Fagundes }
$$
argumentam, ainda, que a essas medidas podem ser associadas outras, como a análise periódica das condições de trabalho, a promoção de educação permanente in loco sobre os riscos ocupacionais e a criação de um canal de comunicação entre a Comissão Interna de Prevenção de Acidentes (CIPA), o serviço de saúde ocupacional e a unidade.

\section{CONSIDERAÇÕES FINAIS}

Os achados desta revisão possibilitam um vislumbre sobre a problemática do absenteísmo entre a equipe de enfermagem lotada em UTI e PS, destacando-se a maior associação de ausências não previstas entre o pessoal de nível médio e entre aqueles que trabalham à noite. A principal causa das ausências foi o adoecimento, sobretudo por distúrbios osteomusculares e transtornos mentais e comportamentais. Destacam-se ainda os efeitos negativos do absenteísmo sobre a organização e a qualidade da assistência prestada ao paciente.

Contudo, devido ao delineamento transversal da maior parte dos artigos desta revisão, além da ausência de estudos de coorte e de caso-controle entre ele, tais achados não fornecem evidência conclusiva sobre o fenômeno nas unidades em questão.

Além disso, carece-se de estudos sobre a temática nas unidades de assistência ao paciente crítico, que abordem aspectos relacionados aos determinantes e consequências do absenteísmo, tais como 
satisfação no trabalho, comprometimento organizacional, oportunidade de ascensão, estresse ocupacional, autonomia, rotina e processo de trabalho, dentre outros.

Sendo assim, pesquisas futuras devem ser conduzidas, de modo a tornar possível o fornecimento de informações passíveis de generalização à sociedade e às organizações de saúde, como forma de orientar o desenvolvimento de intervenções que visem resolver este problema, a fim de promover impacto positivo sobre a qualidade de vida no trabalho desses trabalhadores e de melhorar a qualidade da assistência de enfermagem prestada ao paciente internado nesses setores.

\section{REFERÊNCIAS}

ABREU, R. M. D.; SIMÕES, A. L. A. Ausências por adoecimento na equipe de enfermagem de um hospital de ensino. Ciênc. Cuid. Saúde, Maringá, v. 8, n. 4, p. 637-44, 2009.

\section{ALDERSON, M. Work structure in the} chronic care setting: possible consequences for nurses' mental health. Can. J. Nurs. Res., Montreal, v. 40, n. 3, p. 160-78, 2008.

BARBOZA, D. B.; SOLER, Z. A. S. G. Afastamentos do trabalho na enfermagem: ocorrências com trabalhadores de um hospital de ensino. Rev. Latino-Am. Enfermagem, Ribeirão Preto, v. 11, n. 2, p. 177-83, 2003.
CAMPOS, E. C.; JULIANI, C. M. C. M.; PALHARES, V. C. O absenteísmo da equipe de enfermagem em unidade de pronto socorro de um hospital universitário. Rev. Eletr. Enf. [Internet], v. 11, n. 2, p. 295-302. Disponível em:

http://www.fen.ufg.br/revista/v11/n2/v11n2a0 9.htm. Acesso em: 10 de julho de 2013.

CARNEIRO, T. M.; FAGUNDES, N. C. Absenteísmo entre trabalhadoras de enfermagem em unidade de terapia intensiva de hospital universitário. Rev. Enferm. UERJ, Rio de Janeiro, v. 20, n. 1, p. 84-9, 2012.

CARVAlHO, C. C.; MAGALHÃES, S. R. Síndrome de Burnout e suas consequências nos profissionais de enfermagem. Revista da Universidade Vale do Rio Verde, Três Corações, v. 9, n. 1, p. 200-10, 2011. CASTRO, I.; BERNARDINO, E.; RIBEIRO, E. L. Z. Absenteísmo na enfermagem em UTI neonatal: perfil do profissional e motivos das ausências. Cogitare Enferm., Curitiba, v. 13, n. 3, p. 374-9, 2008.

COSTA, F. M.; VIEIRA, M. A.; SENA, R. R. Absenteísmo relacionado à doenças entre membros da equipe de enfermagem de um hospital escola. Rev. Bras. Enferm., Brasília, v. 62, n. 1, p. 38-44, 2009. 
DAVEY, M. M. et al. Predictors of nurse absenteeism in hospitals: a systematic review. J. Nurs. Manag., Oxford, v. 17, n. 3, p. 31230, 2009.

DUARTE, N. S.; MAURO, M. Y. C. Análise dos fatores de riscos ocupacionais do trabalho de enfermagem sob a ótica dos enfermeiros. Rev. Bras. Saúde Ocup., São Paulo, v. 35, n. 121, p. 157-67, 2010.

FAKIH, F. T.; TANAKA, L. H.; CARMAGNANI, M. I. S. Ausências dos colaboradores de enfermagem do prontosocorro de um hospital universitário. Acta Paul. Enferm., São Paulo, v. 25, n. 3, p. 37885, 2012.

FERREIRA, R. C. et al. Abordagem multifatorial do absenteísmo por doença em trabalhadores de enfermagem. Rev. Saúde Pública, São Paulo, v. 46, n. 2, p. 259-68, 2012.

GAUDINE, A. et al. Effects of absenteeism feedback and goal-setting interventions on nurses' fairness perceptions, discomfort feelings and absenteeism. J. Nurs. Manag., Oxford, v. 21, p. 591-602, 2013.

INOUE, K. C. et al. Absenteísmo-doença da equipe de enfermagem em unidade de terapia intensiva. Rev. Bras. Enferm., Brasília, v. 61, n. 2, p. 209-14, 2008.
INOUE, K. C.; MATSUDA, L. M.; SILVA, D. M. P. P. Absenteísmo em unidade de terapia intensiva de um hospital-escola. Ciênc. Cuid. Saúde, Maringá, v. 7, n. supl.1, p. 11-7, 2008.

IZU, M. et al. Trabalho noturno como fator de risco na carcinogênese. Cienc. Enferm., Concepcion, v. XVII, n. 3, p. 83-95, 2011.

LIMA, A. F. C.; KURGANCT, P. Indicadores de qualidade no gerenciamento de recursos humanos em enfermagem. Rev. Bras.

Enferm., Brasília, v. 62, n. 2, p. 234-9, 2009.

MAGALHÃES, A. M. M.; DALL'AGNOL, C. M.; MARCK, P. B. Carga de trabalho da equipe de enfermagem e segurança do paciente - estudo com método misto na abordagem ecológica restaurativa. Rev. Latino-Am. Enfermagem, Ribeirão Preto, v. 21, n. spe, p. 146-54, 2013.

MAGALHÃES, N. A. C. et al. O absenteísmo entre trabalhadores de enfermagem no contex to hospitalar. Rev. Enferm. UERJ, Rio de Janeiro, v. 19, n. 2, p. 224-30, 2011. RAUHALA, A. et al. What degree of work overload is likely to cause increased sickness absenteeism among nurses? Evidence from the RAFAELA patient classification system. J. Adv. Nurs., Oxford, v. 57, 286-95, 2007. 
SANCINETTI, T. R. et al. Absenteísmo -

doença na equipe de enfermagem: relação

com a taxa de ocupação. Rev. esc. enferm.

USP, São Paulo, v. 43, n. spe2, p. 1277-83,

2009.

SANCINETTI, T. R. et al. Taxa de

absenteísmo da equipe de enfermagem como

indicador de gestão de pessoas. Rev. esc.

enferm. USP, São Paulo, v. 45, n. 4, p. 1007-

12, 2011.

STATISTICS CANADA. Labour Force

Survey. Average Days Lost for Personal

Reasons per Full-Time Employee by

National Occupational Classification for

Statistics (NOC-S) Sex and Age Group,

Annual $(3,4)$. Ottawa: Statistics Canada,

2007. 